\title{
Digital Dark Nudge: An Exploration of When Digital Nudges Unethically Depart
}

\author{
Francis Joseph Costello \\ Sungkyunkwan University \\ joe.costehello@gmail.com
}

\author{
Jin Ho Yun \\ Sungkyunkwan University \\ jin.ho.yun90@gmail.com
}

\author{
Kun Chang Lee \\ Sungkyunkwan University \\ kunchanglee@gmail.com
}

\begin{abstract}
Digital nudging in information systems has become widely prevalent to guide consumers during online decision-making. However, while nudging is about improving the decisions and behaviors in various domains, limited research has explored when digital nudges unethically depart from their intended purpose, whereby opt-in favors profit motives over the user's best interests. In e-commerce, we defined this as a digital dark nudge (DDN) and explored its use in multiple scenarios against a typical shopping experience. Using an online experiment, we study the economic intentions and emotional perceptions of DDNs, while also accounting for impulsiveness as a moderating personality trait. This study first attempts to use priming and status quo bias as a theoretical lens. Empirical results show increasing evidence of the perverse effects of using DDNs in online ecommerce whereby consumers revert to their status quo, less likelihood of purchase. Our results provide further warning to practitioners about their use of ethical practices such as digital nudging.
\end{abstract}

\section{Introduction}

As online shopping becomes more ubiquitous with younger shoppers, time spent on digital platforms has created new opportunities for online sellers. Nevertheless, have you ever considered why sometimes you get annoyed by some online purchase experiences? For example, imagine yourself wanting to purchase an airplane ticket. An initial discount is offered to you; once you proceed past the first trivial pages, you find a charge of $\$ 5$ has been added for seat selection. You did not initially opt into this selection. You then find yourself going back and opting out of the charge by finding a small tick box obscured from sight with a small print that is hard to read. This is an example of when digital nudging unethical departs.

Influenced by Behavioral Economics on how people make decisions, Thaler and Sunstein [1] introduced the idea of nudging to understand how decision-making may deviate from rationality [2]. Defined as "any aspect of the choice architecture that alters people's behavior in a predictable way without forbidding any option or significantly changing their economic incentives," [1] nudges help re-arrange choices in a way that nudges individuals into making more desired economic decisions [2,3]. Since its inception, nudges have also been deployed in the digital world, known as "digital nudging" [4,5]. However, the more technology has become ubiquitous, the more boundaries between the digital and physical worlds have blurred [3]. Thus, the need for investigation into a greater understanding of digital nudging has increased [6]. This notion has led IS scholars to increasingly see digital nudging as a promising research field [7].

The application of digital nudging has found success in a variety of settings. Most notably, a review by Caraban et al. [8] found they were effectively used to promote health and physical activity and sustainable behaviors such as recycling. Other successes are found in increasing human performance through improved recall or reduction of information overload. Lastly, digital nudges have been successfully used to strengthen privacy and security, such as improving password security [3]. Despite these positive implementations, ethical concerns on the use of digital nudges have been raised in academia [9-11]. By nature, digital nudges were designed to shape and change user's behavior for the better. Nonetheless, just like it is possible to predict people's behavior by understanding their psychological principles and adjusting them for good [6], predicting behavioral outcomes for the betterment of the designer's desired goals is just as easy.

Based on cleverly manipulated UX design of online platforms, such hijacking of the known irrational nature of human decision-making has led to research investigating what is known as a dark pattern [12]. Dark patterns have been identified and discussed much in the UX design field [6,13-15]. Currently, it is understood that over ten types exist and are used commonly [13], and research has started to delve into understanding their effects on users. Such investigation has led to several papers investigating users' ability to identify dark patterns and investigated their perceptions $[6,16,17]$. However, the identification of dark patterns showed mixed findings. 
For instance, Bhoot et al. [6] showed that some dark patterns were identified with $70 \%$ or more accuracy, whereas others were identified less than $50 \%$ of the time. Nonetheless, when participants were confronted about their use, perceptions were almost always negative $[16,17]$.

Till now, dark patterns have been explored in the context of e-commerce twice. First, Costello et al. [18] explored the dark pattern hidden costs in a pilot study, whereas Bhoot et al. [6] asked participants to experience forced continuity on Audible's website. Both studies showed that dark patterns were detrimental by significantly reducing purchasing and recommendation intention [18] and increasing frustration [6]. These preliminary studies have provided evidence of the potential effects of dark patterns used within e-commerce, yet only tested one type of dark pattern with small samples. This is problematic as the use of dark patterns in e-commerce is more pervasive than initially thought [13], leaving open questions about other types and individual effectiveness when used within e-commerce. Another limitation seen within these two prior studies is their lack of a theoretical understanding of the notions at play. Thus, this study looks to rectify this by examining the use of dark patterns and their effects on consumer's intentions by employing priming and status quo theory.

In this paper, we undertook an online experiment on 121 people. We prepared five unexplored categories of dark patterns identified in Mathur et al. [13], creating two scenarios for each. Our results show that the main effects hold and provide ample evidence on the actual effects of DDNs on consumers' shopping experiences and intentions. Based on our evidence, we validate with greater confidence the original proposition seen in prior work and attempt to provide a theoretical proposition of the underlying processes that are manifesting in consumers when confronted with dark patters within online commerce.

The remainder of this paper is structured in the following way. First, a theoretical proposition of the use of dark patterns in online commerce is proposed and how these represent a departed digital nudge (DDN) from the status quo expectations of consumers. Next, the methodological approach is presented before analytical results are explored. Lastly, a discussion is made.

\section{Literature review}

\subsection{Digital nudges and dark patterns}

Following the first conceptualization of a digital nudge in 2016 [4], digital nudging has been explored in numerous empirical papers in various settings [19-24]. Defined as "the use of user-interface design elements to guide people's behavior in digital choice environments" [4], digital nudging follows the same principles as nudge theory. One of these, opt-out as opposed to opt-in, means choice architecture is designed to automatically opt people into a decision based on the predicted rational choice that benefits that person $[1,25]$. Within e-commerce, digital nudging was first explored through the lens of numeric and semantic priming [19]. This research found that such priming was effective in an auction-type scenario but ineffective for fixed-price selling. The results of this study show a glimpse of the potential effects digital nudging can have on consumers and their willingness to pay based on choice architecture design manipulation.

Dennis et al. [19] demonstrated that digital nudging could be very effective when executed well. However, such efficacy has also led to ethical concerns and manipulating such knowledge for perverse incentives. Despite work on providing ethical guidance on the use of digital nudges by Lembcke et al. [11], Meske and Amojo [10], Renaud and Zimmermann [9], and Fansher et al. [15], evidence continues to show the unethical use of choice architecture design is being used [13]. Known as a dark pattern within the UX field, evidence of their existence and effects on people are slowly simmering to the surface within academia. For instance, Nouwens et al. [17] identified consent pop-ups that used dark patterns and found that user's intentions did not match their ideal privacy settings when shown their decisions. Furthermore, Di Geronimo et al. [16] found that dark patterns in mobile applications were so common that participants in the experiments could not detect them. Lastly, Bhoot et al. [6] found a positive correlation between the frequency of occurrence of a dark pattern with a participant's trustworthiness and level of frustration.

The study of dark patterns in e-commerce has been made under different lenses. For example, Moser et al. [26] analyzed leading e-commerce websites and found dark patterns that induce impulse buying. Similarly, Mathur et al. [13] investigated the prominence of dark patterns in e-commerce, finding that $11 \%$ of e-commerce websites used some form of dark pattern out of a sample of $11 \mathrm{k}$ websites.

In this study, we adopt five different dark patterns for analysis within this paper. Specifically, these included: sneak in the basket, hidden subscription, urgency, confirmshaming, and hard to cancel. Next, we will present each definition of a dark pattern adapted from Mathur et al. [13]. 
Sneak in the basket is where sellers add products/services into a user's shopping cart without their prior consent or knowledge. These are often promoted as 'bonus' and /or 'necessary' products.

Hidden subscription is when the user is charged a recurring fee after they are led to believe a one-off charge or free trial was being provided. This is usually seen within service products where addons are common.

Urgency is a signal that allows the user to know a deadline for the sale or potential purchase of an item. This usually makes discounts and offers more desirable. However, evidence has shown that these are usually not used honestly and are repeated regularly on different days [13].

Confirmshaming was first coined on darkpatterns.org [12] and uses emotive language to steer consumers into a particular choice. These are usually shown after the consumer has decided to cancel or not proceed with a purchase. Alternatively, they appear when an action, such as providing an email, is not made. At this point, a pop-up box will show the consumer a message that consists of shameful traits such as not being rich. For instance, confirmshaming uses sarcasm to make the person think about their current financial situation shamefully: "No thanks, I am already rich and do not need this offer."

Hard to cancel is a dark pattern that makes a particular action harder than it should be. This has the goal of dissuading users from taking any action against the will of the seller. These tactics usually make it easy for users to sign up for a subscription or membership while making it hard to cancel afterward. Furthermore, information is usually hidden at the sign-up stage on how you have to cancel after signing up.

The first attempts to analyze consumers' perceptions of dark patterns in e-commerce were investigated by Costello et al. [18] and Bhoot et al. [6]. Both studies showed the detrimental effects of a dark pattern by reducing purchasing and recommendation intention [18] and increasing distrust and frustration in the shopping experience [6]. Despite the merits of these papers, their sample size was small, and they only tested one type of dark pattern within their work. In this paper, we first attempt to explore the economic intentions of consumers when confronted with a dark pattern within e-commerce. The following section defines our proposition as well as explores its theoretical basis within the context of e-commerce.

\subsection{Digital dark nudge}

In this section, drawing from research into priming and status quo bias theory, we postulate a theoretical framework to understand consumers' perceptions and economic intentions when faced with a digital nudge and their unethical counterpart, referred to here as a digital dark nudge (DDN). As a digital nudge observes the same ethical rules first proposed in nudge theory $[1,25]$, any e-commerce that adheres to these ethical principles is a digital nudge in the plain sense [4]. However, when similar design features are used in the choice architecture for nefarious purposes, the process departs from what can be defined as a digital nudge and thus becomes a DDN.

A DDN is not a digital nudge. By its very definition, a digital nudge cannot be unethical [1], and hence a DDN can only be viewed as a nefarious clone. It is neither just a dark pattern. Dark patterns are the specific UI design features used, whereby much research has produced good taxonomies [13,27]. Within e-commerce and following early attempts by Costello et al. [18] and Bhoot et al. [6], we define a DDN as the overall process that starts with a primed perceived benefit and then departs from a digital nudge when a dark pattern is applied to the choice architecture at a later payment stage. A DDN is then the conscious decision of the consumer to depart from the payment process (revert to the status quo) as the initially perceived benefits are no longer maintained to consumers in the later process when opt-in dark patterns are embedded.

In this paper, we propose that within the realm of e-commerce, researchers move to a more holistic approach in which the analysis starts with priming and moves through the UI field's dark patterns before reaching the effects on consumer's decision-making. Such attempts require a theoretical foundation that can effectively understand the response to dark pattern stimuli. This is where priming and status quo bias is utilized (see Figure 1).

\subsubsection{Priming}

As first presented in the setting of e-commerce by Dennis et al. [19], numeric and semantic priming are considered stimuli used to influence specific cognition or behavior from a person [28]. Further, as this study is limited to an online setting, we adopt the definition of concurrent priming. In other words, the priming takes place on the same screen of the shopping experience itself [19] rather than in a prior setting. The initial process starts with a perceived benefit when it comes to a digital dark nudge (using dark patterns). The perceived benefit is cognitively primmed into the consumer and preludes the focal purpose of online selling a product/service [28,29]. People will be unconscious "fished" into following this perceived benefit (e.g., a perceived discount) before the DDN 
starts to begin later in the payment process; thus, we present the following proposition:

Axiom 1: For both a digital nudge and a digital dark nudge, a perceived benefit will be presented through priming in the initial stage of the process.

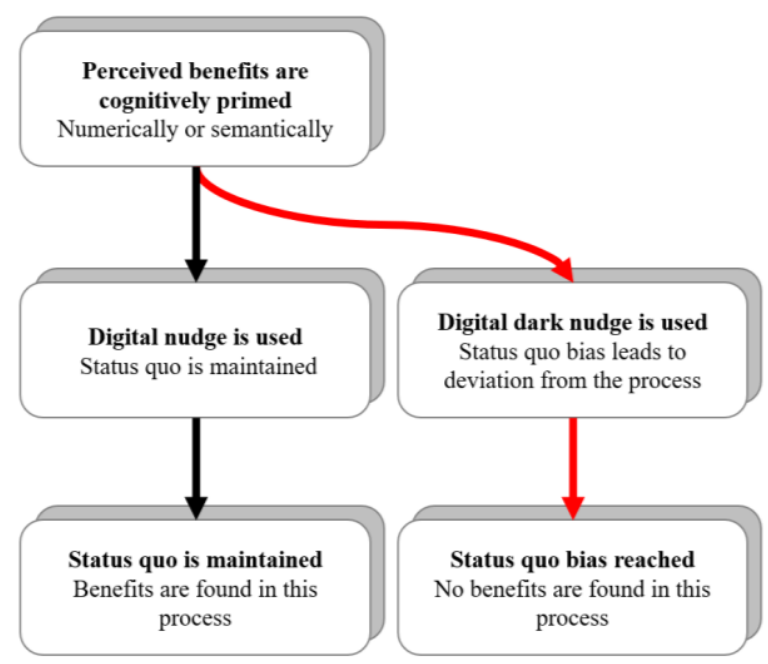

Figure 1. A theoretical model of a DDN

\subsubsection{Status quo bias}

As consumers progress through the shopping process, they continuously update their perceptions of the overall process and act accordingly. Research has shown that at any point, if someone feels that a risky decision is present, they are more likely to stick to the status quo [30]. Such decision-making in individuals has consistently been shown whereby disadvantages of leaving an offer loom larger than advantages presented, leading to a status quo bias [31]). For instance, research has shown that the SQB exists even in decision contexts in which the status quo option is unambiguously less attractive than the alternatives [32] or whereby inertia of the alternative is salient in the decision process [33]. SQB is so consistent that research into the neural underpinning has now been identified in regions of the brain [34].

In the case of a DDN, if a consumer is given a choice whereby they have automatically been opted into a purchase decision they did not priorly want or agree to, then the seeming advantages that were initially presented no longer hold. When the perceived benefit becomes distorted or unwanted by the consumer, people will bias the original benefit (i.e., status quo). Suppose we revert to the example provided in the introduction. At the point the consumer sees an extra charge they were unaware of, in that case, a status quo bias will become more salient. Reasons for this are when the consumer must change the default (opt-in) seat selection, making the consumer uneasy, it leads to a potentially negative emotional response about the process. Furthermore, research has consistently shown that a negative shopping experience that induces a negative feeling will lead to less likelihood of the consumer recommending this product/service [35]. Therefore, we hypothesize:

H1a: Consumers will be more risk-averse when confronted with a digital dark nudge and will not proceed with a purchase.

H1b. Consumers confronted with a digital dark nudge will show a lower intention to recommend using the website to friends and family.

\subsection{Emotional states and impulsiveness}

The connection between emotion and behavior has a long history in consumer behavior research. Extant literature tells us that positive moods appear to show a greater likelihood that behaviors with more positive associations will be acted upon [36]. Furthermore, the link between emotion and impulse buying behavior has also been found [37]. For example, in a field study, it was found that positive emotions affected ones' shopping enjoyment and increased the urge to buy impulsively. On the other hand, the authors found evidence that a negative emotion did not influence consumers' impulse buying urges [38]. Similar findings have been found within online shopping too. Results based on website design showed that emotional cues could positively influence the likelihood and magnitude of impulse buying. In other words, impulse buying can be maximized when positive affective reactions alongside positive cognitive reactions are simultaneously maximized on a website design [39].

Concerning this study and as previously hypothesized, we believe that the experience of a DDN will have a negative impact on consumers. As seen in this prior literature, if a negative experience is provided, it will lead to negative emotions and reduce the likelihood of impulsive buying [18]. Thus, if a person demonstrates a more impulsive attitude and enjoys a positive affective experience in online shopping, we can predict that they will be more open to acting upon the impulse to proceed with a purchase. Therefore, within our model, the consumer's emotion can be seen as manipulating one's affective state, leading to the moderation of impulsiveness on the final purchase intention and recommendation intention. Thus, we hypothesize the following: 
H2a. Consumers confronted with a digital dark nudge will have negative emotions after experiencing one.

$H 2 b$. Impulsiveness will moderate the effect of digital nudges on purchase and recommendation intention.

\section{Methodology}

Participants $(N=120$; collected on Prolific.co; mean age $=30 ; 56 \%$ women $)$ were randomly assigned to one of two between-subjects conditions: the DDN condition versus a typical shopping experience. The participant's pool was selected among the relatively young consumers from Prolific since they are more likely to have an online shopping experience than those older consumers.

Five dark patterns were used for analyzing the DDN hypotheses (refer to section 2.1.). Two hypothetical shopping scenarios were then created for each, making a total of ten scenarios for participants to see. The scenarios were selected to gender-neutral items, and that was not too cheap nor too expensive. These included: electronics, airplane tickets, brain game applications, music and movie subscription, and unisex sunglasses. These five types of DDN will represent the most significant number of actual instances on the websites. Some of those represented cognitive biases (i.e., sunk cost fallacy, scarcity bias, framing effect, or none) [13].

In the DDN condition, participants were provided with a budget in mind (ranging from $\$ 25$ to \$700) and told that they had found the right website for the product/service they wanted. Next, they were put

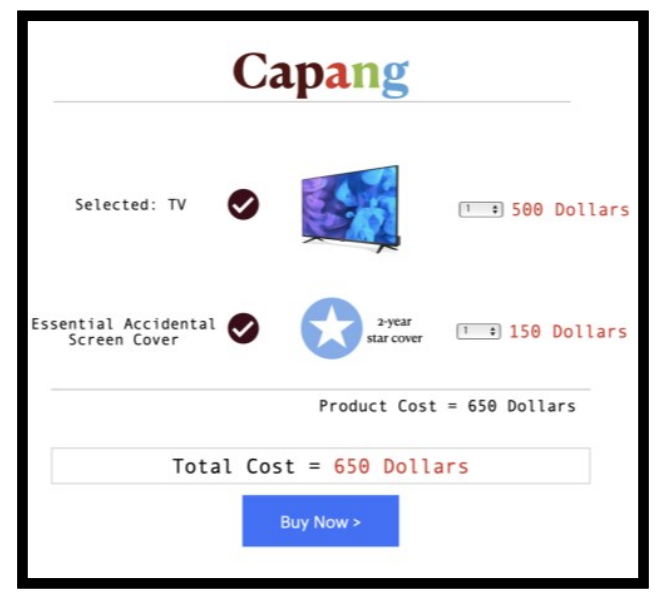

(a) A dark pattern through $1 / 2$ slides of context, whereby a page break and action were placed to create the online experience of pressing a button to continue to the next page. Words such as "Proceed" or "Buy now" were placed between stimuli to create a more realistic scenario. The final page always provided the final checkout page and did not have an action to do as the participants would answer questions based on their intentions and experience of this website up till this point.

Following [18], we adopted and adapted a similar line of questioning and employed one-way ANOVA for measuring stand-alone questions about the two dependent variables and emotion: purchase intention: How likely would you proceed with this purchase? (measured on a 9-Likert scale; 1 = very unlikely, $9=$ highly likely); recommendation intention: How likely would you recommend the use of this website to friends and family? (measured on a 9-Likert scale; 1 = very unlikely, $9=$ highly likely). Next, the participants were presented with an adapted version of the SAM test [34] which examined their valence and arousal after experiencing the online website $(1=$ low levels, $5=$ no valence/arousal, $9=$ high levels).

The typical shopping experience condition included the same protocol seen within the DDN condition. However, all the DDN manipulations were removed (see Figure 2 for an example comparison). The same budget and scenario were provided as seen in the DDN. Furthermore, the order of the questions asked remained the same. Both conditions were randomized within each condition to remove any potential learning of a particular category.

Lastly, before collecting demographic data, we measured our moderator's extent of each participant's impulsiveness. Specifically, we used the Barrat

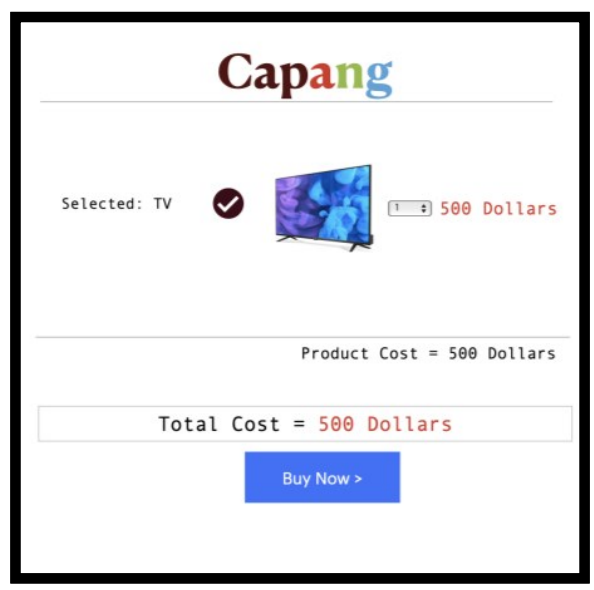

(b) Typical Shopping Experience

Figure 2. A comparison between (a) a DDN and (b) a typical shopping experience 
Impulsive Scale [41], whereby participants were presented with 30 items seen in the original test. Participants were asked on a four-point scale (Rarely/never; Occasionally; Often; Almost Always/Always). The rationale for the moderator is as follows: if a consumer likes the experience of the digital platform and feels the deal is good, within their interest to buy, and has no underlying tricks, they will most likely consider going through with the transaction. If a consumer is also more impulsive, this intention will be much higher. Conversely, if the consumer doubts about the offer or service being provided, this will likely hinder any impulsive temptations and help remove any irrational decisions. Thus, consumers with online experience will show less impulsiveness when they experience the DDN condition.

\section{Results}

We performed the analyses based on the average of all five categories. Our central hypotheses $1 \mathrm{a}$ and $1 \mathrm{~b}$ stated that consumers would be less likely to show purchase intentions and recommendations to others in the DDN conditions than those in the typical shopping experience. Therefore, we conducted one-way ANOVA (Analysis of Variance) on purchase intentions and recommendations to others (i.e., our main dependent variables).

In average, the main effects revealed significant differences between two conditions on two separate dependent variables. Participants reported lower scores on purchase intention for the DDN $(\mathrm{M}=5.18)$ than for the typical shopping experience $(\mathrm{M}=5.85$, $\mathrm{F}(1,119)=8.423, \mathrm{p}=.004)$. They also reported lower scores on recommending to others for the DDN (M = 4.99) than for the typical shopping experience $(\mathrm{M}=$ $5.66, \mathrm{~F}(1,119)=6.894, \mathrm{p}=.010)$. Further, we found that participants reported lower scores on a valence measure $(\mathrm{M}=5.24)$ for the DDN than for the typical shopping experience $(\mathrm{M}=5.83, \mathrm{~F}(1,119)=10.521$, $\mathrm{p}$ $=.002)$. They also reported lower scores on a arousal measure $(\mathrm{M}=4.45)$ for the DDN than for the typical shopping experience $(\mathrm{M}=5.48, \mathrm{~F}(1,119)=8.895$, $\mathrm{p}$ $=.003)$.

We also explored each digital nudging category. In the sneaking type, participants reported lower scores on purchase intention for the DDN $(\mathrm{M}=4.94)$ than for the typical shopping experience $(\mathrm{M}=6.20$, $\mathrm{F}(1,119)=17.674, \mathrm{p}=.000)$. They also reported lower scores on recommending to others for the DDN (M = 4.71) than for the typical shopping experience $(\mathrm{M}=$ $6.00, F(1,119)=15.813, p=.000)$. Further, we found that participants reported lower scores on a valence measure $(\mathrm{M}=5.02)$ for the DDN than for the typical shopping experience $(\mathrm{M}=6.23, \mathrm{~F}(1,119)=21.624, \mathrm{p}$ $=.000)$. They also reported lower scores on a arousal measure $(\mathrm{M}=4.29)$ for the DDN than for the typical shopping experience $(\mathrm{M}=5.41, \mathrm{~F}(1,119)=14.183, \mathrm{p}$ $=.000)$.

In the hidden subscription type, participants reported lower scores on purchase intention for the DDN $(\mathrm{M}=5.10)$ than for the typical shopping experience $(\mathrm{M}=6.24, \mathrm{~F}(1,119)=15.824, \mathrm{p}=.000)$. They also reported lower scores on recommending to others for the DDN $(\mathrm{M}=5.00)$ than for the typical shopping experience $(\mathrm{M}=5.91, \mathrm{~F}(1,119)=8.930, \mathrm{p}$ $=.003)$. Further, we found that participants reported lower scores on a valence measure $(M=5.03)$ for the DDN than for the typical shopping experience $(\mathrm{M}=$ $6.05, F(1,119)=17.286, p=.000)$. They also reported marginally lower scores on a arousal measure $(\mathrm{M}=$ 4.48) for the DDN than for the typical shopping experience $(\mathrm{M}=5.08, \mathrm{~F}(1,119)=3.664, \mathrm{p}=.058)$.

In the urgency type, participants reported lower scores on purchase intention for the DDN $(M=5.22)$ than for the typical shopping experience $(M=6.08$, $\mathrm{F}(1,119)=7.168, \mathrm{p}=.000)$. They also reported lower scores on recommending to others for the DDN (M = 5.08) than for the typical shopping experience $(\mathrm{M}=$ $5.74, \mathrm{~F}(1,119)=4.523, \mathrm{p}=.036)$. Further, we found that participants reported lower scores on a valence measure $(\mathrm{M}=5.50)$ for the DDN than for the typical shopping experience $(\mathrm{M}=6.01, \mathrm{~F}(1,119)=4.279, \mathrm{p}$ $=.041)$. However, they reported similar scores on a arousal measure $(\mathrm{M}=4.99)$ for the DDN than for the typical shopping experience $(\mathrm{M}=5.32, \mathrm{~F}(1,119)=$ $1.310, \mathrm{p}=.255)$.

Participants reported similar scores on all of the variables in the last two types (i.e., confirmshaming and hard-to-cancel type). In addition, there were no significant differences between the two nudging conditions on two dependent variables and SAM measures $(\mathrm{p}>.05)$.

In addition, we regressed two digital nudging conditions on two dependent variables (i.e., purchase intention and recommendation to others), and their interactions, respectively. For the purchase intentions, although the analysis showed a significant main effect of nudging conditions $(\beta=0.703, \mathrm{t}(117)=3.012, \mathrm{p}$ $=.003)$, there were no significant main effect of impulsiveness as well their interactions $(p>.05)$. Still, since impulsiveness is the continuous measure, we explored the interaction further using the JohnsonNeyman floodlight technique (see Figure 3) [42]. This results revealed a positive and significant effect of nudging conditions on purchase intentions for degrees of impulsiveness (mean-centered) greater than -0.17 but less than $0.56\left(\beta_{\mathrm{JN}}=2.97, \mathrm{SD}=.28, \mathrm{p}=0.05\right)$. For the recommendation to others, although the analysis 
showed a significant main effect of nudging conditions $(\beta=0.682, \mathrm{t}(117)=2.668, \mathrm{p}=.009)$, there were no significant main effect of impulsiveness as well their interactions $(p>.05)$. Similarly, we also explored the interaction further using the Johnson-Neyman floodlight technique [42]. This results revealed a positive and significant effect of nudging conditions on purchase intentions for degrees of impulsiveness (mean-centered) greater than -0.08 but less than 0.69 $\left(\beta_{\mathrm{JN}}=23.35, \mathrm{SD}=.28, \mathrm{p}=0.05\right)$.

\section{Discussion}

This study has explored the concept of a digital dark nudge (DDN) within e-commerce. A DDN is the study of dark patterns used within e-commerce website design to trick consumers into opting into purchases and subscriptions that they may not be fully aware. Thus, this paper sought to extend and analyze the use of dark patterns in e-commerce $[6,18]$ by providing a more holistic view through the theoretical lens of priming [19] and status quo bias theory [30]. Compared with much of the current research on dark patterns $[6,16,17]$, a study of a DNN attempts to analyze consumers' economic and emotional responses to them specifically. Furthermore, due to the known interactions between emotion and impulsiveness [37-39], the personality trait of impulsiveness as an interaction effect with the primary dependent variables was also explored.

Five categories of DDNs were explored. Each was explored through two main dimensions: economic and emotion. From an economic standpoint, we found strong evidence of the resistance to the sneaking, hidden subscription, and urgency DDNs, allowing us to accept $\mathrm{H} 1 \mathrm{a}$ and $\mathrm{H} 1 \mathrm{~b}$ for these categories. Our evidence showed that consumers did not perceive the original benefit to hold as the process continued, thus showing that they showed status quo bias towards the initial offer or no purchase. This finding was not found in confirmshaming and hard to cancel DDNs. We suggest this holds because opted-in items were easily calculated compared with the initial offer for sneaking and hidden subscriptions. For urgency, this effect is potentially holding as consumers did not like the time pressure, which was opted into after seeing the offer whereby no mention of scarcity was initiated. As confirmshaming and hard to cancel are not financially quantifiable, it is feasible to say that their effect did not hold. This is because e-commerce is a financial transaction, and thus semantic tricks may not directly influence economic decisions. In addition, we found that recommendations were significantly higher amongst participants that were not exposed to the DDN. This is consistent with research finding that positive experiences will lead to a recommendation [35].

Our obtained results also allowed us to accept $\mathrm{H} 2 \mathrm{a}$ based on the analysis of both consumer's valance and arousal after experiencing each condition. Significant findings were found for the same three categories. Consistent with prior findings [36], including Bhoot et al. [6], who showed negative emotions were induced through frustration when participants were presented with a dark pattern. As seen for sneaking, hidden subscription, and urgency, reduced economic intentions were first displayed, followed by more negative emotions towards the process. Such notions have been corroborated in which negative feelings can result from maintaining the current state (i.e., not purchasing) under uncertainty [43]. Once again, the lack of financial risks presented with the confirmshaming and hard to cancel categories may have contributed to their insignificance. It is worth noting that it does not mean that these two categories do not have an effect. It may just be that the context was not effectively executed, or alternatively, their effects are more pronounced in other IS systems, such
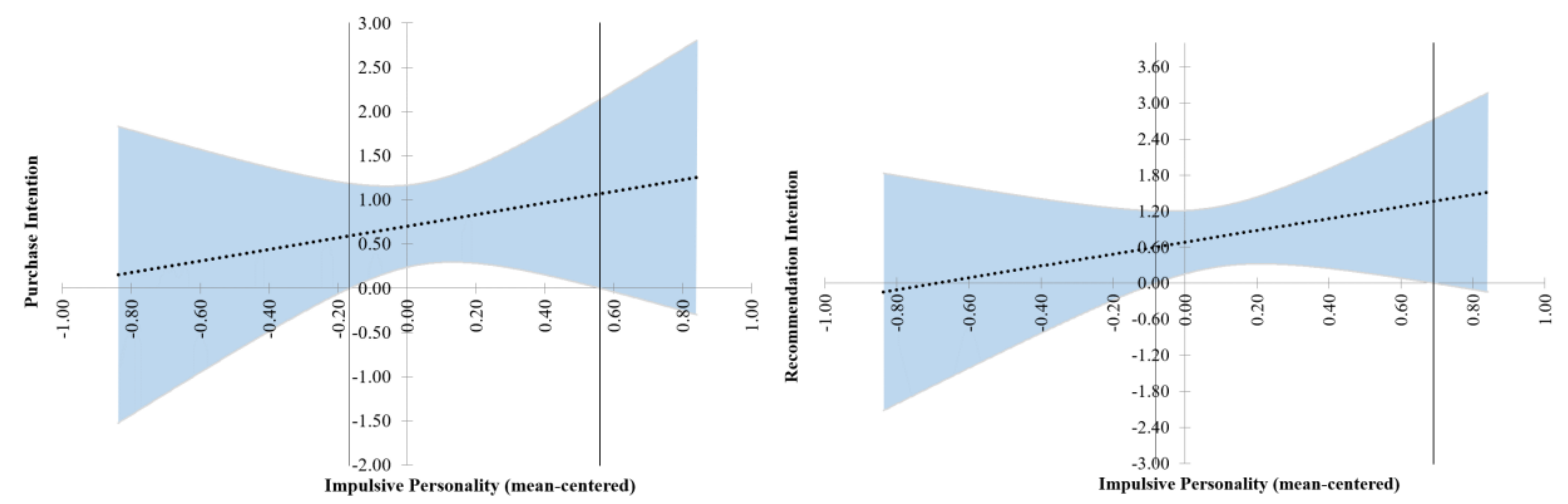

Figure 3. The moderating effect of impulsive personality 
as security, where finances are not primarily necessary.

Next, H2b was rejected. However, due to the nature of impulsiveness being a continuous variable, the Johnson-Neyman floodlight technique [42] was employed to find any moderating interaction when the data were mean-centered. This further analysis found some proclivities for the interaction effect of impulsiveness, suggesting that it is an area of interest that needs further analysis.

In summary, this study provides some significant theoretical and practical contributions to digital nudging and ethics in UX design. Firstly, this paper is the first attempt to understand the use of unethical digital nudge-like choice architecture through the lens of priming and status quo bias theory. As many websites bid to gain consumers' attention online, attention-grabbing tactics are more widely being used. Although most are in good faith, we propose that this is used as priming in the case of a DDN [19]. Once the consumer is primmed or "fished" from the online marketplace, the following stages can effectively begin for the DDN. Thus, we believe that the use of priming can effectively explain the first stage of a DDN.

Next, we show that non-transparent, opt-in features that favor the seller's profit motives can negatively impact consumers' perceptions of that shopping process, leading to status quo bias. Therefore, this paper has shown early empirical evidence of the effects that DDNs are having in forcing consumers to revert to a status quo. Contrary to nudge theory that attempts to aid consumers in making a favorable rational decision based on an optin criterion, DDNs' opt-in features make consumers feel uneasy and thus push them to decide in favor of the status quo (i.e., no purchase). Such findings expand our knowledge of status quo bias theory within the realm of DDN and show how status quo bias can be an effective lens to view consumers' decisionmaking processes within the broader field of digital nudging.

From a practical standpoint, our evidence suggests that the use of DDNs is not in the best interest of consumers, potentially in the long run, not in the sellers' best interest. In the short term, they may be able to profit from myopic tendencies in all consumers. However, if persisted, they may lose the custom of those consumers and thus damage the brand's reputation. Thus, practitioners should take seriously the ethical concerns put forward in previous digital nudging papers so that they can avoid any bad will with their consumers.

\section{Limitations and future research}

To conclude this paper, we must address the limitations of this work and provide future research opportunities. Firstly, a significant limitation lies in only assessing five categories of dark patterns. Prior research has found over ten variations of dark patterns [13], and manipulating and empirically assessing these will be crucial in understanding their effects on consumers and thus validating the DDN concept put forward in this paper. This is important because, as seen within this paper, the main effect was significant. However, when this analysis was broken into each category, we found that two categories were insignificant. This means that the manipulation of these DDNs was not correctly implemented or did not entirely affect the initially hypothesized consumers. Thus, it will be crucial for future work to analyze these further to help understand their actual effects. Another limitation was in the results of the hypothesized moderating effect. As an interaction effect was not significant, this will need to be further elucidated in future studies with other potential moderators (e.g., an individual online experience of how much they frequently access to or spend time on online webpages or visual allocation clusters [44]).

Furthermore, it leads to further questions about what might also be at play when consumers are confronted with a DDN. For example, could the potential habituation effect help uncover unknown possibilities that influence a DDN? The habituation effect in users' reactions and interpretations of specific IS stimuli has previously been discovered [45]. Thus, it is not impossible to believe that a similar effect may be at play here.

Acknowledgment: This work was supported by the National Research Foundation of Korea(NRF) grant funded by the Korea government(MSIT) (No. 2020R1F1A1074808).

\section{References}

[1] R.H. Thaler, C.R. Sunstein, Nudge: Improving decisions about health, wealth, and happiness, 1st Editio, Penguin, Michigan, 2008.

[2] A. Caraban, L. Konstantinou, E. Karapanos, The nudge deck: A design support tool for technologymediated nudging, in: DIS 2020 - Proceedings of the 2020 ACM Designing Interactive Systems Conference, Association for Computing Machinery, Inc, 2020: pp. 395-406. 
[3] V. Zimmermann, K. Renaud, The nudge puzzle: Matching nudge interventions to cybersecurity decisions, ACM Transactions on Computer-Human Interaction. 28 (2021).

[4] M. Weinmann, C. Schneider, J. vom Brocke, Digital Nudging, Business and Information Systems Engineering. 58 (2016) 433-436.

[5] C. Schneider, M. Weinmann, J. vom Brocke, Digital Nudging: Guiding Online User Choices through Interface Design, Communications of the ACM. 61 (2018) 67-73.

[6] A.M. Bhoot, M.A. Shinde, W.P. Mishra, Towards the identification of dark patterns: An analysis based on end-user reactions, in: ACM International Conference Proceeding Series, Association for Computing Machinery, 2020: pp. 24-33.

[7] T. Mirsch, C. Lehrer, R. Jung, Making Digital Nudging Applicable: The Digital Nudge Design Method, in: Proceedings of the 39th International Conference on Information Systems (ICIS), AIS Electronic Library, San Francisco, 2018.

[8] A. Caraban, E. Karapanos, D. Gonçalves, P. Campos, 23 Ways to Nudge: A review of technologymediated nudging in human-computer interaction, in: Conference on Human Factors in Computing Systems - Proceedings, Association for Computing Machinery, 2019.

[9] K. Renaud, V. Zimmermann, Ethical guidelines for nudging in information security \& privacy, International Journal of Human Computer Studies. 120 (2018) 22-35.

[10] C. Meske, I. Amojo, Ethical Guidelines for the Construction of Digital Nudges, Proceedings of the 53rd Hawaii International Conference on System Sciences. 3 (2020) 3928-3937.

[11] T.-B. Lembcke, N. Engelbrecht, A.B. Brendel, L. Kolbe, To nudge or not to nudge: Ethical considerations of digital nudging based on its behavioral economics roots, in: ECIS 2019 Proceedings, 2019: pp. 1-17.

[12] H. Brignull, A. Darlo, What Are Dark Patterns?, (2019). https://www.darkpatterns.org/ (accessed March 12, 2020).

[13] A. Mathur, G. Acar, M.J. Friedman, E. Lucherini, J. Mayer, M. Chetty, A. Narayanan, Dark patterns at scale: Findings from a crawl of $11 \mathrm{~K}$ shopping websites, Proceedings of the ACM on Human-Computer Interaction. 3 (2019).
[14] C.M. Gray, Y. Kou, B. Battles, J. Hoggatt, A.L. Toombs, The dark (patterns) side of UX design, in: Conference on Human Factors in Computing Systems - Proceedings, Association for Computing Machinery, 2018.

[15] M. Fansher, S.S. Chivukula, C.M. Gray, \#Darkpatterns: UX Practitioner Conversations About Ethical Design, in: Conference on Human Factors in Computing Systems - Proceedings, Association for Computing Machinery, 2018.

[16] L. di Geronimo, L. Braz, E. Fregnan, F. Palomba, A. Bacchelli, UI Dark Patterns and Where to Find Them: A Study on Mobile Applications and User Perception, in: Conference on Human Factors in Computing Systems - Proceedings, Association for Computing Machinery, 2020.

[17] M. Nouwens, I. Liccardi, M. Veale, D. Karger, L. Kagal, Dark Patterns after the GDPR: Scraping Consent Pop-ups and Demonstrating their Influence, in: Conference on Human Factors in Computing Systems - Proceedings, Association for Computing Machinery, 2020.

[18] F.J. Costello, J.H. Yun, K.C. Lee, A NeuroIS Investigation of the Effects of a Digital Dark Nudge, in: F.D. Davis, R. Riedl, J. vom Brocke, P.-M. Léger, A.B. Randolph, T. Fischer (Eds.), Lecture Notes in Information Systems and Organisation, Springer International Publishing, Cham, 2020: pp. 64-70.

[19] A.R. Dennis, L. (IVY) Yuan, X. Feng, E. Webb, C.J. Hsieh, Digital Nudging: Numeric and Semantic Priming in E-Commerce, Journal of Management Information Systems. 37 (2020) 39-65.

[20] N. Huang, P. Chen, Y. Hong, S. Wu, Digital Nudging for Online Social Sharing: Evidence from A Randomized Field Experiment, Proceedings of the 51st Hawaii International Conference on System Sciences. 9 (2018) 1483-1491.

[21] S. Kankane, C. DiRusso, C. Buckley, Can we nudge users toward better password management? An initial study, Conference on Human Factors in Computing Systems - Proceedings. 2018-April (2018) $1-6$.

[22] D. Schneider, T. Grupp, S. Lins, A. Benlian, A. Sunyaev, Nudging Users Into Online Verification: The Case of Carsharing Platforms, ICIS 2017 Proceedings. (2017) 1-20.

[23] C. Stryja, G. Satzger, V. Dorner, A decision support system design to overcome resistance towards sustainable innovations, ECIS 2017 Proceedings. 2017 (2017) 2885-2895. 
[24] N. Székely, M. Weinmann, J. vom Brocke, Nudging people to pay $\mathrm{Co} 2$ offsets-The effect of anchors in flight booking processes, ECIS 2016 Proceedings, 2016.

[25] R.H. Thaler, The Power of Nudges, for Good and Bad, The New York Times. 26 (2015) 1-5. http://www.nytimes.com/2015/11/01/upshot/thepower-of-nudges-for-good-andbad.html\%5Cnhttp://www.nytimes.com/2015/11/01/u pshot/the-power-of-nudges-for-good-andbad.html?rref=upshot\&_r=2.

[26] C. Moser, S.Y. Schoenebeck, P. Resnick, Impulse buying: Design practices and consumer needs, in: Conference on Human Factors in Computing Systems - Proceedings, Association for Computing Machinery, 2019.

[27] A. Mathur, J. Mayer, What makes a dark pattern... dark? design attributes, normative considerations, and measurement methods, in: Conference on Human Factors in Computing Systems - Proceedings, Association for Computing Machinery, 2021.

[28] J.A. Bargh, M.J. Ferguson, Beyond behaviorism: On the automaticity of higher mental processes, Psychological Bulletin. 126 (2000) 925-945.

[29] Dennis, Fuller, Valacich, Media, Tasks, and Communication Processes: A Theory of Media Synchronicity, MIS Quarterly. 32 (2008) 575.

[30] W. Samuelson, R. Zeckhauser, Status quo bias in decision making, Journal of Risk and Uncertainty. 1 (1988) 7-59.

[31] D. Kahneman, J.L. Knetsch, R.H. Thaler, Anomalies: The Endowment Effect, Loss Aversion, and Status Quo Bias, Journal of Economic Perspectives. 5 (1991) 193-206.

[32] G. Suri, G. Sheppes, C. Schwartz, J.J. Gross, Patient Inertia and the Status Quo Bias: When an Inferior Option Is Preferred, Psychological Science. 24 (2013) 1763-1769.

[33] J. Nel, C. Boshoff, Status quo bias and shoppers' mobile website purchasing resistance, European Journal of Marketing. 54 (2020) 1433-1466.

[34]S.M. Fleming, C.L. Thomas, R.J. Dolan, Overcoming status quo bias in the human brain, Proceedings of the National Academy of Sciences of the United States of America. 107 (2010) 6005-6009.

[35] W. Messner, Understanding the influence of culture on customer engagement and recommendation intentions, Journal of Strategic Marketing. (2020).
[36] M.P. Gardner, Mood States and Consumer Behavior: A Critical Review, Journal of Consumer Research. 12 (1985) 281.

[37] P. Weinberg, W. Gottwald, Impulsive consumer buying as a result of emotions, Journal of Business Research. 10 (1982) 43-57.

[38] S.E. Beatty, M. Elizabeth Ferrell, Impulse buying: Modeling its precursors, Journal of Retailing. 74 (1998) 161-167.

[39] D.V. Parboteeah, J.S. Valacich, J.D. Wells, The influence of website characteristics on a consumer's urge to buy impulsively, Information Systems Research. 20 (2009) 60-78.

[40] M.M. Bradley, P.J. Lang, Measuring emotion: The self-assessment manikin and the semantic differential, Journal of Behavior Therapy and Experimental Psychiatry. 25 (1994) 49-59.

[41] J.H. Patton, M.S. Stanford, E.S. Barratt, Factor structure of the barratt impulsiveness scale, Journal of Clinical Psychology. 51 (1995) 768-774.

[42] S.A. Spiller, G.J. Fitzsimons, J.G. Lynch, G.H. Mcclelland, Spotlights, Floodlights, and the Magic Number Zero: Simple Effects Tests in Moderated Regression, Journal of Marketing Research. 50 (2013) 277-288.

[43] D. Kahneman, A. Tversky, The Psychology of Preferences, 246 (1982) 160-173.

[44] X. Gong, Webpage human computer interface design, in: 2010 International Conference on Multimedia Technology, ICMT 2010, 2010.

[45] B.B. Anderson, J.L. Jenkins, A. Vance, C.B. Kirwan, D. Eargle, Your memory is working against you: How eye tracking and memory explain habituation to security warnings, Decision Support Systems. 92 (2016) 3-13. 